\title{
From nonalcoholic fatty liver disease to metabolic-asso- ciated fatty liver disease: Big wave or ripple?
}

\author{
Seong Hee Kang ${ }^{1, *}$, Yuri Cho ${ }^{2,3, *}$, Soung Won Jeong ${ }^{4, *}$, Seung Up Kim, ${ }^{5,6}$, Jin-Woo Lee ${ }^{7}$; and on behalf of Korean \\ NAFLD Study Group
}

'Department of Internal Medicine, Yonsei University Wonju College of Medicine, Wonju; ${ }^{2}$ Department of Internal Medicine, CHA Gangnam Medical Center, CHA University School of Medicine, Seoul; ${ }^{3}$ Center for Liver and Pancreatobiliary Cancer, National Cancer Center, Goyang; ${ }^{4}$ Department of Internal Medicine, Soonchunhyang University Seoul Hospital, Soonchunhyang University College of Medicine, Seoul; ${ }^{5}$ Department of Internal Medicine, Yonsei University College of Medicine, Seoul; ' Yonsei Liver Center, Severance Hospital, Seoul; ${ }^{7}$ Department of Internal Medicine, Inha University Hospital, Inha University School of Medicine, Incheon, Korea

There is some dissatisfaction with the term "nonalcoholic fatty liver disease (NAFLD)," which overemphasizes alcohol and underemphasizes the importance of metabolic risk factors in this disease. Recently, a consensus recommended "metabolic (dysfunction)-associated fatty liver disease (MAFLD)" as a more appropriate term to describe fatty liver diseases (FLD) associated with metabolic dysfunction. During the definition change from NAFLD to MAFLD, subjects with FLD and metabolic abnormalities, together with other etiologies of liver diseases such as alcohol, virus, or medication who have been excluded from the NAFLD criteria, were added to the MAFLD criteria, while subjects with FLD but without metabolic abnormality, who have been included in the NAFLD criteria, were excluded from the MAFLD criteria. This means that there is an emphasis on the metabolic dysfunction in MAFLD which may underestimate the prognostic value of hepatic steatosis itself, whereas the MAFLD criteria might better identify subjects who are at a higher risk of hepatic or cardiovascular outcomes. However, non-metabolic risk NAFLD subjects who are excluded from the MAFLD criteria are missed from the diagnosis, and their potential risk can be the cause of future diseases. Although huge controversies remain, this review focused on summarizing recent studies that compared the clinical and prognostic characteristics between subjects with NAFLD and MAFLD. (Clin Mol Hepatol 2021;27:257-269)

Keywords: Non-alcoholic fatty liver disease; Metabolic (dysfunction)-associated fatty liver disease; Outcome; Metabolic syndrome; Diabetes mellitus

\begin{abstract}
Abbreviations:
ALT, alanine aminotransferase; APRI, aspartate aminotransferase to platelet ratio index; AST, aspartate aminotransferase; BMI, body mass index; $\mathrm{Cl}$, confidence interval; CVD, cardiovascular disease; FIB-4, fibrosis-4; FLD, fatty liver disease; $H R$, hazard ratio; MAFLD, metabolic-associated fatty liver disease; NAFLD, nonalcoholic fatty liver disease; NHANES, National Health and Nutrition Examination Survey; MR, metabolic risk; T2DM, type 2 diabetes mellitus
\end{abstract}

\author{
Corresponding author : Seung Up Kim \\ Department of Internal Medicine, Yonsei University, College of Medicine, \\ 50-1 Yonsei-ro, Seodaemun-gu, Seoul 03722, Korea \\ Tel: +82-2-2228-1944, Fax: +82-2-393-6884 \\ E-mail:ksukorea@yuhs.ac \\ https://orcid.org/0000-0002-9658-8050

\section{Jin-Woo Lee} \\ Department of Internal Medicine, Inha University Hospital, Inha University \\ School of Medicine, 27 Inhang-ro, Jung-gu, Incheon 22332, Korea \\ Tel: +82-32-890-2548, Fax: +82-32-890-2549 \\ E-mail:jin@inha.ac.kr \\ https://orcid.org/0000-0002-7227-4938
}

*Seong Hee Kang, Yuri Cho, and Soung Won Jeong equally contributed to this work as co-first authors. 


\section{INTRODUCTION}

The term "nonalcoholic fatty liver disease (NAFLD)" was first introduced by Schaffner and Thaler' in 1986 to describe fatty liver disease (FLD) occurring in the absence of significant alcohol intake. This characterization overemphasizes the absence of alcohol use and underemphasizes the importance of metabolic risk factors that fuel the progression of liver disease in NAFLD. ${ }^{2}$ In addition, many criticisms have focused on the fact that NAFLD may trivialize these diseases, reducing their consideration in the public health policy.

To date, it has become clear that NAFLD is a heterogeneous disorder with different metabolic and genetic factors involved in its pathogenesis contributing to its progression and prognosis., ${ }^{3,4}$ The heterogeneous pathogenesis of NAFLD and inaccuracies in its definition necessitates a review of its nomenclature. ${ }^{5}$ In 2019, Eslam et al. ${ }^{2}$ provided suggestions on alternative terminologies that more accurately reflect the pathogenesis of this disease, which might help in patient stratification. Experts reached a consensus that NAFLD does not reflect our current understanding of this disease, and metabolic (dysfunction)-associated fatty liver disease (MAFLD) might be a more appropriate overarching term. ${ }^{2}$

MAFLD represents a hepatic manifestation of systemic metabolic dysfunction. MAFLD also warrants a "positive" diagnosis, negating a diagnosis based on the exclusion of other concomitant liver diseases. This novel nomenclature may be useful for clinical trials and research studies. In addition, the role of MAFLD in patients with concomitant liver disease has been recognized. However, since this change has not been fully accepted by major societies, future studies are required to confirm the feasibility of this novel terminology and to reach a consensus on the MAFLD criteria. Moreover, non-metabolic risk (MR) NAFLD subjects who are excluded from MAFLD criteria are missed from the diagnosis, prompting caution in establishing follow-up strategy and assessing the prognosis.

Our primary aim was not to review the recent studies on MAFLD without comparison to NAFLD, as the significant overlap between MAFLD and NAFLD might add only a few insights into the current literature. Instead, we focused on summarizing recent studies that compared the clinical and prognostic characteristics between subjects with NAFLD and those with MAFLD, which might be helpful in deciding the use of optimal terminology in current clinical practice.

\section{DIAGNOSTIC CRITERIA OF NAFLD AND MAFLD}

NAFLD is defined by the 1) evidence of hepatic steatosis, either by imaging or histology, demonstrating the presence of steatosis in $>5 \%$ of the hepatocytes, and the 2) lack of secondary causes of hepatic fat accumulation, such as significant alcohol consumption (daily alcohol consumption $\geq 30 \mathrm{~g}$ for men and $\geq 20 \mathrm{~g}$ for women), long-term use of a steatogenic medication, or monogenic hereditary disorders (Table 1). ${ }^{6,7}$ In the majority of patients, NAFLD is commonly associated with metabolic comorbidities such as obesity, diabetes mellitus, and dyslipidemia. ${ }^{5}$

In contrast, the diagnosis of MAFLD is based on the histological (biopsy), imaging, or blood biomarker evidence of fat accumulation in the liver, in addition to one of the following three criteria: overweight/obesity, presence of type 2 diabetes mellitus (T2DM), or evidence of metabolic dysregulation. ${ }^{8}$ The latter is defined by the presence of at least two metabolic risk abnormalities, as listed in Table 1.

Additionally, a panel of international experts have proposed that patients with cirrhosis who fulfill the diagnostic criteria for MAFLD should be considered as having MAFLD-related cirrhosis; therefore, the term "cryptogenic cirrhosis" should be avoided in this group. ${ }^{8}$ The diagnostic criteria for MAFLD-related cirrhosis include patients with cirrhosis in the absence of typical histological signs suggestive of steatohepatitis who fulfill at least one of the following: past or present evidence of metabolic risk factors that meet the criteria to diagnose MAFLD (Table 1), together with at least one of the following: 1) documentation of MAFLD on a previous liver biopsy, or 2) historical documentation of steatosis by hepatic imaging (Table 2). In particular, a history of alcohol intake should be considered, as patients may have dual disease etiology in the presence of alcohol use disorders. ${ }^{8}$

\section{ADDED POPULATIONS ACCORDING TO MAFLD CRITERIA}

A panel of international experts proposed a new definition for the diagnosis of MAFLD based on the presence of metabolic dysfunction and not the absence of other conditions. ${ }^{8}$ As a result, there is no reference to alcohol consumption or viral hepatitis in the MAFLD criteria, and this means that MAFLD can coexist with other liver diseases. ${ }^{2}$ Added population according to MAFLD criteria included subjects who fulfilled the definition of MAFLD, but not the NAFLD criteria. The subjects in this specific population 
Table 1. Diagnostic criteria for NAFLD and MAFLD

\begin{tabular}{|c|c|}
\hline NAFLD $^{6,7}$ & MAFLD $^{8}$ \\
\hline \multirow{2}{*}{$\begin{array}{l}\text { For defining NAFLD, there must be } \\
\text { 1) evidence of hepatic steatosis, either by imaging or } \\
\text { histology (steatosis in }>5 \% \text { of hepatocytes), and } \\
\text { 2) lack of secondary causes of hepatic fat accumulation } \\
\text { such as significant alcohol consumption (a daily } \\
\text { alcohol consumption } \geq 30 \mathrm{~g} \text { for men and } \geq 20 \mathrm{~g} \text { for } \\
\text { women), long-term use of a steatogenic medication, } \\
\text { or monogenic hereditary disorders }\end{array}$} & $\begin{array}{l}\text { In adults with hepatic steatosis (detected either by imaging techniques, blood } \\
\text { biomarkers/scores, or liver histology), these three groups are classified as MAFLD } \\
\text { 1) Overweight/obesity (defined as BMI } \geq 25 \mathrm{~kg} / \mathrm{m}^{2} \text { in Caucasians or BMl } \geq 23 \mathrm{~kg} / \mathrm{m}^{2} \\
\text { in Asians) } \\
\text { 2) Lean/normal weight (defined as BMI }<25 \mathrm{~kg} / \mathrm{m}^{2} \text { in Caucasians or BMl }<23 \mathrm{~kg} / \mathrm{m}^{2} \\
\text { in Asians) }\end{array}$ \\
\hline & $\begin{array}{l}\text { The presence of at least two of the following metabolic risk abnormalities: } \\
\text { - Waist circumference } \geq 102 / 88 \mathrm{~cm} \text { in Caucasian men and women (or } \geq 90 / 80 \\
\mathrm{~cm} \text { in Asian men and women) } \\
\text { - Blood pressure } \geq 130 / 85 \mathrm{mmHg} \text { or specific drug treatment } \\
\text { - Plasma triglycerides } \geq 150 \mathrm{mg} / \mathrm{dL}(\geq 1.70 \mathrm{mmol} / \mathrm{L} \text { ) or specific drug treatment } \\
\text { - Plasma HDL-cholesterol }<40 \mathrm{mg} / \mathrm{dL}(<1.0 \mathrm{mmol} / \mathrm{L} \text { ) for men and }<50 \mathrm{mg} / \mathrm{dL} \\
(<1.3 \mathrm{mmol} / \mathrm{L} \text { ) for women or specific drug treatment } \\
\text { - Prediabetes (i.e., fasting glucose levels } 100 \text { to } 125 \mathrm{mg} / \mathrm{dL}[5.6 \text { to } 6.9 \mathrm{mmol} / \mathrm{L}] \text {, } \\
\text { or 2-hour post-load glucose levels } 140 \text { to } 199 \mathrm{mg} / \mathrm{dL}[7.8 \text { to } 11.0 \mathrm{mmol} \text { or } \\
\text { HbA1c } 5.7 \% \text { to } 6.4 \% \text { [39 to } 47 \mathrm{mmol} / \mathrm{mol} \text { ) } \\
\text { - Homeostasis model assessment of insulin resistance score } \geq 2.5 \\
\text { - Plasma high-sensitivity C-reactive protein level }>2 \mathrm{mg} / \mathrm{L}\end{array}$ \\
\hline
\end{tabular}

3) Type 2 diabetes mellitus (according to widely accepted international criteria)

NAFLD, nonalcoholic fatty liver disease; MAFLD, metabolic-associated fatty liver disease; BMI, body mass index; HDL, high-density lipoprotein.

Table 2. Diagnostic criteria for MAFLD-related cirrhosis ${ }^{8}$

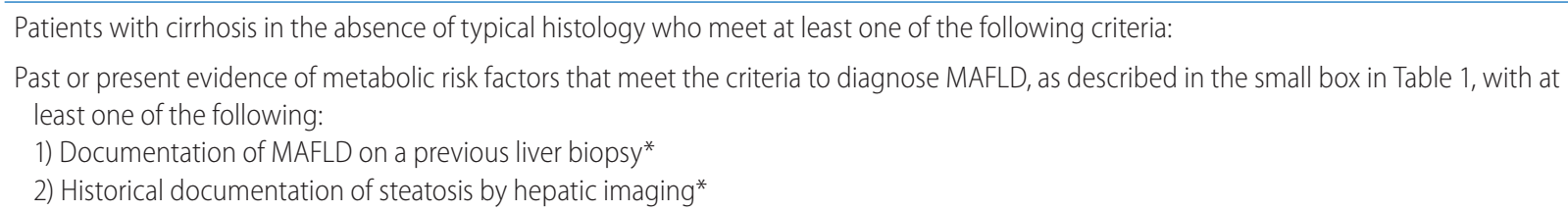

MAFLD, metabolic-associated fatty liver disease.

*History of past alcohol intake should be considered as patients may have a dual disease etiology with alcohol use disorder.

showed FLD and metabolic abnormalities, together with other etiologies of liver diseases such as alcohol, virus, or secondary causes including steatogenic medication or hereditary disorders (Fig. 1), who were excluded from the NAFLD criteria.

In a recent study by Zheng et al. focused on the PERSONS cohort; ${ }^{9}$ and of the 780 consecutively enrolled subjects with liver biopsy, $663(85 \%)$ fulfilled both the NAFLD and MAFLD criteria. $^{10}$ The difference in the number of subjects between the two groups (670 NAFLD vs. 773 MAFLD) was due to seven patients with FLD who were lean by body mass index (BMI) without satisfying the metabolic risk threshold (defined as non-MR NAFLD), and the inclusion of 110 patients with FLD and other etiologies of liver diseases (66 with excess alcohol consumption, 40 with viral hepatitis, and four with autoimmune hepatitis). Notably, age, sex, comorbid diseases (i.e., diabetes mellitus and hypertension), variant distribution of the PNPLA3 gene, metabolic profile, and histol- ogy, including liver steatosis and fibrosis, were not significantly different between the two groups. In a subgroup analysis of the MAFLD group, patients with MAFLD and significant alcohol consumption presented with significantly higher gamma-glutamyl transferase levels and exhibited more severe liver steatosis compared to the pure MAFLD (single etiology) group.

In another recent Taiwanese study by Huang et al.," 175 patients with histology-verified liver steatosis and 10 with cryptogenic cirrhosis were classified according to the diagnostic criteria of MAFLD and NAFLD. A total of 76 patients (41.1\%) presented as both NAFLD and MAFLD, and nine patients (4.9\%) had non-MR NAFLD, 81 patients (43.8\%) had MAFLD without NAFLD, and the remaining 19 patients (10.3\%) did not meet the criteria for either category. Although there was no significant difference in age or sex between the two groups, BMI was significantly higher in patients with MAFLD without NAFLD compared to the non-MR 


\section{CLINICAL and MOLECULAR}

\section{HEPATOLOGY}

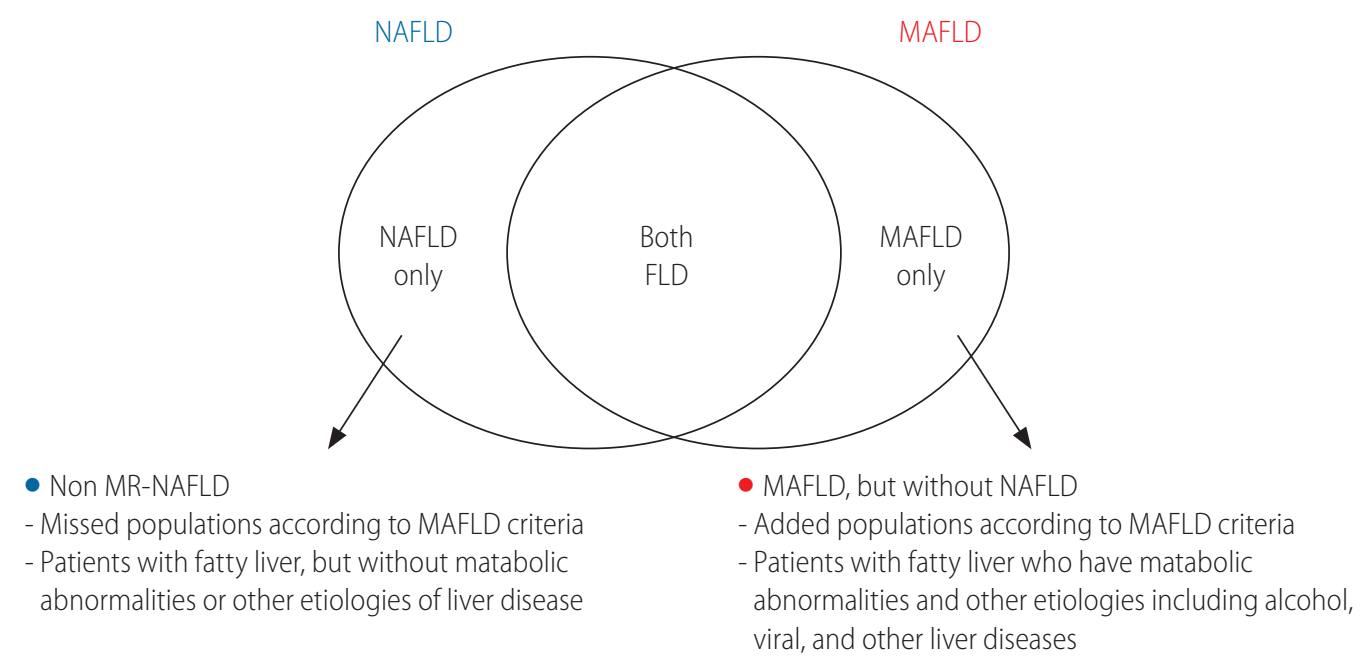

Figure 1. Venn diagram highlighting the similarities and differences between NAFLD and MAFLD. NAFLD, nonalcoholic fatty liver disease; MAFLD, metabolic-associated fatty liver disease; FLD, fatty liver disease; MR, metabolic risk.

NAFLD group (27.31 vs. $\left.21.36 \mathrm{~kg} / \mathrm{m}^{2} ; P<0.001\right)$. Among the 81 patients with MAFLD without NAFLD, 35 (43.2\%) presented with hepatitis B virus infection, 23 (28.4\%) were taking culprit medications, $10(12.3 \%)$ had hepatitis C virus infection, seven (8.6\%) presented with autoimmune liver disease, five $(6.2 \%)$ had chronic alcoholism, and one (1.2\%) was positive for hepatitis $\mathrm{E}$ virus infection. This group also included 19 patients (23.5\%) with liver cirrhosis, 30 (37.0\%) with hypertension, 26 (32.1\%) with T2DM, and $16(19.8 \%)$ with dyslipidemia, while none of these comorbidities were found in the non-MR NAFLD group. Moreover, patients with MAFLD, but without NAFLD, showed a significant increase in disease severity, as evaluated by both histology and laboratory findings, compared to those with non-MR NAFLD. Hepatitis B virus infection, hypertension, and T2DM were also independently associated with advanced fibrosis in patients with MAFLD.

In the most recent cross-sectional study of the National Health and Nutrition Examination Survey (NHANES) of the general population of the USA, the prevalence of NAFLD and MAFLD were similar in the 1,710 subjects at $37.1 \%$ and $39.1 \%$, respectively. ${ }^{12}$ Only 13 subjects with NAFLD did not meet the MAFLD criteria (non-MR NAFLD), and 54 subjects with MAFLD did not meet the NAFLD criteria (MAFLD, but without NAFLD), mainly due to significant alcohol consumption or the presence of viral hepatitis. None of the 13 subjects with non-MR NAFLD presented with advanced liver fibrosis; however, those with MAFLD, but without NAFLD, showed similar advanced fibrosis to the overall estimate. The prevalence of advanced fibrosis among subjects with NAFLD and MAFLD was $7.5 \%$ and $7.4 \%$, respectively. The authors sug- gested that the recent change in diagnostic criteria did not affect the prevalence of the condition in the general USA population. The clinical significance of studies using the MAFLD criteria is summarized in Table 3.

\section{MISSED POPULATIONS WHEN USING MAFLD CRITERIA}

Missed population according to MAFLD criteria includes the subjects who fulfilled the definition of NAFLD, but not MAFLD (subjects with non-MR NAFLD). These subjects present with FLD, but without metabolic abnormalities or other etiologies of liver disease (Fig. 1), while meeting the NAFLD criteria. Although patients with non-MR NAFLD comprise a minority of these populations, many studies comparing the clinical characteristics and long-term outcomes of subjects with non-MR NAFLD to those without NAFLD or MAFLD and those with MAFLD are actively ongoing. Given the ambiguity in the definition of non-MR NAFLD, appropriate therapeutic strategies remain unclear.

A recent study using the NHANES III database, which is a periodic survey conducted by the Centers for Disease Control and Prevention in the USA, focused on the comparison of subjects with MAFLD and those with non-MR NAFLD and severe steatosis. ${ }^{13} \mathrm{Al}-$ though the absolute sample size of the subjects with non-MR NAFLD was extremely small $(n=46)$, comprising only around $1 \%$ of the subjects with MAFLD $(n=4,087)$, and corresponding statistical errors cannot be completely removed, this study offered two 
Table 3. Clinical significance of studies using MAFLD criteria

\begin{tabular}{|c|c|c|c|}
\hline \multirow{2}{*}{$\begin{array}{l}\text { Study } \\
\text { Zheng et al. }{ }^{10}\end{array}$} & \multicolumn{2}{|c|}{ Number of patients } & \multirow{5}{*}{$\begin{array}{l}\text { Clinical significance } \\
\text { 1. Clinical characteristics including metabolic profile, } \\
\text { comorbid disease, and histology were not different } \\
\text { between NAFLD and MAFLD groups. } \\
\text { 2. Patients with dual etiology (MAFLD and alcohol) had } \\
\text { higher gamma-glutamyl transferase levels and exhibited } \\
\text { more severe hepatic steatosis compared to pure MAFLD } \\
\text { group (single etiology). }\end{array}$} \\
\hline & Total/ NAFLD/MAFLD & $780 / 670 / 773$ & \\
\hline & Both NAFLD and MAFLD & 663 & \\
\hline & Non-MR NAFLD & 7 & \\
\hline & MAFLD, but without NAFLD & $110(\mathrm{~A} / 66, \mathrm{~V} / 40, \mathrm{I} / 4)$ & \\
\hline \multirow[t]{4}{*}{ Huang et al. } & Total/ NAFLD/MAFLD & $166 / 85 / 157$ & \multirow{4}{*}{$\begin{array}{l}\text { 1. MAFLD, but without NAFLD group exhibited a significant } \\
\text { increase in disease severity, as evaluated by both histology } \\
\text { and laboratory findings, compared to those with non-MR } \\
\text { NAFLD. } \\
\text { 2. MAFLD criteria included an additional } 38.9 \% \text { of patients } \\
\text { with hepatic steatosis, and it can help identify those with a } \\
\text { high degree of disease severity for early intervention better } \\
\text { than the previous NAFLD criteria. }\end{array}$} \\
\hline & Both NAFLD and MAFLD & 76 & \\
\hline & Non-MR NAFLD & 9 & \\
\hline & MAFLD, but without NAFLD & $81(A / 5, V / 46, M / 23,1 / 7)$ & \\
\hline \multirow{4}{*}{$\begin{array}{c}\text { Ciardullo and } \\
\text { Perseghin }^{12}\end{array}$} & Total/ NAFLD/MAFLD & 728/674/715 & \multirow{4}{*}{$\begin{array}{l}\text { 1. None of the } 13 \text { subjects with non-MR NAFLD presented } \\
\text { with advanced liver fibrosis. However, those with MAFLD, } \\
\text { but without NAFLD, showed similar advanced fibrosis to } \\
\text { the overall estimate. The prevalence of advanced fibrosis } \\
\text { among subjects with NAFLD and MAFLD was } 7.5 \% \text { and } \\
\text { 7.4\%, respectively. } \\
\text { 2. The authors suggested that the recent change in } \\
\text { diagnostic criteria did not affect the prevalence of the } \\
\text { condition in the general USA population }\end{array}$} \\
\hline & Both NAFLD and MAFLD & 661 & \\
\hline & Non-MR NAFLD & 13 & \\
\hline & MAFLD, but without NAFLD & $54(\mathrm{NA})$ & \\
\hline \multirow[t]{4}{*}{ Lee et al. ${ }^{16}$} & Total/ NAFLD/MAFLD & $3,628,540 / 2,680,217 / 3,573,644$ & \multirow{4}{*}{$\begin{array}{l}\text { 1. A considerable proportion of middle-aged Korean adults } \\
\text { have MAFLD, without satisfying the former definition of } \\
\text { NAFLD. The change from NAFLD to MAFLD criteria may } \\
\text { identify a greater number of individuals with metabolically } \\
\text { complicated fatty liver and increased risk for CVD. } \\
\text { 2. These studies might indicate that patients with non-MR } \\
\text { NAFLD should be closely monitored, as they experience an } \\
\text { increased risk for poor outcomes. }\end{array}$} \\
\hline & Both NAFLD and MAFLD & $2,625,321$ & \\
\hline & Non-MR NAFLD & 54,896 & \\
\hline & MAFLD, but without NAFLD & $948,323(\mathrm{NA})$ & \\
\hline \multirow{4}{*}{$\begin{array}{l}\text { Yamamura et } \\
\text { al. } .^{18}\end{array}$} & Total/ NAFLD/MAFLD & $726 / 541 / 609$ & \multirow{4}{*}{$\begin{array}{l}\text { 1. The MAFLD definition better identifies the group with } \\
\text { fatty liver and significant fibrosis evaluated by non-invasive } \\
\text { tests. Moreover, in patients with MAFLD, even mild alcohol } \\
\text { consumption was associated with worsening of hepatic } \\
\text { fibrosis measures. } \\
\text { 2. It might be suggested that subjects with non-MR } \\
\text { NAFLD might have no urgent diagnostic and therapeutic } \\
\text { intervention needs due to a potentially favorable disease } \\
\text { course. }\end{array}$} \\
\hline & Both NAFLD and MAFLD & 424 & \\
\hline & Non-MR NAFLD & 117 & \\
\hline & MAFLD, but without NAFLD & 185 (NA) & \\
\hline \multirow[t]{4}{*}{ Lin et al. ${ }^{22}$} & Total/ NAFLD/MAFLD & $13,083 / 4,347 / 4,087$ & \multirow{4}{*}{$\begin{array}{l}\text { 1. MAFLD were significantly older and had higher BMI and } \\
\text { higher proportions of metabolic comorbidities (T2DM, } \\
\text { hypertension) compared to subjects with NAFLD. } \\
\text { 2. Subjects with MAFLD and alcohol consumption were } \\
\text { younger and had fewer metabolic disorders and a higher } \\
\text { proportion of advanced fibrosis compared to those } \\
\text { without. }\end{array}$} \\
\hline & Both NAFLD and MAFLD & 3,727 & \\
\hline & Non-MR NAFLD & 620 & \\
\hline & MAFLD, but without NAFLD & 360 (NA) & \\
\hline
\end{tabular}


Table 3. Continued

\begin{tabular}{|c|c|c|c|}
\hline \multirow{3}{*}{$\begin{array}{l}\text { Study } \\
\text { Wai-Sun Wong } \\
\text { et al. }^{23}\end{array}$} & \multicolumn{2}{|c|}{ Number of patients } & \multirow{5}{*}{$\begin{array}{l}\text { Clinical significance } \\
\text { 1. The prevalence of NAFLD and MAFLD was } 25.7 \% \text { and } \\
25.9 \% \text {, respectively, and the addition of the MAFLD criteria } \\
\text { did not significantly change the prevalence of NAFLD. } \\
\text { 2. However, the incidence of MAFLD was } 25 \% \text { lower than } \\
\text { that of NAFLD. This difference was predominantly observed } \\
\text { in subjects with a BMI of }<23 \mathrm{~kg} / \mathrm{m}^{2} \text {. This data suggests } \\
\text { that the new MAFLD criteria may exclude patients with a } \\
\text { more benign clinical course. }\end{array}$} \\
\hline & Total/ NAFLD/MAFLD & $277 / 261 / 263$ & \\
\hline & Both NAFLD and MAFLD & 247 & \\
\hline & Non-MR NAFLD & 14 & \\
\hline & MAFLD, but without NAFLD & $16(\mathrm{~A} / 3, \mathrm{~V} / 13)$ & \\
\hline \multirow[t]{4}{*}{ Niriella et al. ${ }^{24}$} & Total/ NAFLD/MAFLD & 1028/940/990 & \multirow{4}{*}{$\begin{array}{l}\text { 1. Although NAFLD and MAFLD had similar metabolic traits } \\
\text { at baseline and similar outcomes after 7-years, the MAFLD } \\
\text { but without NAFLD group seemed to have higher risk of } \\
\text { adverse outcomes compared to the non-MR NAFLD group. } \\
\text { Although the increase in the index population was small, } \\
\text { redefining NAFLD as MAFLD seemed to improve clinical } \\
\text { utility. }\end{array}$} \\
\hline & Both NAFLD and MAFLD & 902 & \\
\hline & Non-MR NAFLD & 38 & \\
\hline & MAFLD, but without NAFLD & 88 (NA) & \\
\hline
\end{tabular}

MAFLD, metabolic-associated fatty liver disease; NAFLD, nonalcoholic fatty liver disease; MR, metabolic risk; A, alcohol consumption; V, viral hepatitis; I, autoimmune hepatitis; M, medication; NA, not available; CVD, cardiovascular disease; BMI, body mass index; T2DM, type 2 diabetes mellitus.

important clinical implications. First, although most metabolic parameters, including BMI, uric acid, lipid levels, HbA1c, and fasting sugar, were significantly lower in subjects with non-MR NAFLD and severe steatosis compared to those with MAFLD, their liver enzyme levels were statistically comparable (mean $24.00 \mathrm{IU} / \mathrm{L}$ in non-MR NAFLD and severe steatosis vs. $23.96 \mathrm{IU} / \mathrm{L}$ in MAFLD for alanine aminotransferase [ALT] level, $P=0.990$; mean $27.63 \mathrm{IU} / \mathrm{L}$ in non-MR NAFLD and severe steatosis vs. $25.35 \mathrm{IU} / \mathrm{L}$ in MAFLD for aspartate aminotransferase [AST] level, $P=0.340$ ). In addition, the degree of fibrotic burden, assessed by AST to platelet ratio index (APRI) and fibrosis-4 (FIB-4) index, was statistically similar between the two groups (mean 0.38 in non-MR NAFLD and severe steatosis vs. 0.26 in MAFLD for $A P R I, P=0.275$; mean 1.25 in non-MR NAFLD and severe steatosis vs. 1.06 in MAFLD for FIB-4, $P=0.486)$. Therefore, the authors insisted that the degree of liver damage is similar between subjects with MAFLD and those with non-MR NAFLD and severe steatosis who cannot be classified as MAFLD. This means that, despite their relative rarity, subjects with non-MR NAFLD should not be ignored; rather, more attention is needed for accurate assessment and appropriate therapeutic interventions in clinical practice. Second, this study highlighted that subjects with non-MR NAFLD and severe steatosis were significantly younger than those with MALFD (mean 40 vs. 48 years). To date, the unfavorable influence of hepatic steatosis on longterm outcomes, such as the increased risk of developing cardiovascular disease (CVD) events and T2DM, have been well reported. ${ }^{14,15}$ In addition, hepatic steatosis can develop as the first manifestation of overt metabolic dysfunction, and increased age is a significant predictor of metabolic syndrome. These facts indicate that regular monitoring is needed for younger subjects with non-MR NAFLD and severe steatosis, who currently do not show metabolic dysfunction but may develop various metabolic phenotypes, including MAFLD. In another recent study conducted in South Korea, the proportion of subjects with non-MR NAFLD was only $0.6 \% .{ }^{16}$ Although the comparison between subjects with non-MR NAFLD and those with MAFLD was not feasible, the adjusted risk of CVD in subjects with non-MR NAFLD was slightly increased with statistical significance (hazard ratio [HR], 1.09; 95\% confidence interval [Cl], 1.03-1.15) compared to subjects without NAFLD or MAFLD. Among the surrogates of CVD, the risk of myocardial infarction specifically increased (HR, 1.14). These studies might indicate that patients with non-MR NAFLD should be closely monitored, as they experience an increased risk for poor outcomes.

In contrast, a recent Chinese study using the NHANES III database showed that the proportion of subjects with non-MR NAFLD was higher $(6.3 \% ; n=793$ of 12,571$)$ in these populations, and that the subjects presented with favorable renal outcomes compared to those with MAFLD (6.93\% vs. $14.02 \%$ for albumin-tocreatinine ratio; mean 83.49 vs. $74.06 \mathrm{~mL} / \mathrm{min} / 1.73 \mathrm{~m}^{2} ; 6.43 \%$ vs. $20.28 \%$ for stage $3-5$ chronic kidney disease; all $P<0.05){ }^{17}$ In another study from Japan, the proportion of non-MR NAFLD was $15.3 \%$ and MAFLD without NAFLD was $24.2 \%$ among the subjects with FLD. ${ }^{18}$ Group comparison showed significant elevations 
Table 4. Comparisons of the severity of hepatic steatosis and fibrosis between non-overlapping NAFLD and MAFLD groups

\begin{tabular}{|c|c|c|c|}
\hline Surrogates & Non-MR NAFLD $(n=117)$ & MAFLD, but without NAFLD $(n=185)$ & $P$-value \\
\hline Fatty liver index & $6(3$ to 11$)$ & 50 (32 to 71$)$ & $<0.001$ \\
\hline APRI & $0.2(0.2$ to 0.3$)$ & $0.3(0.2$ to 0.4$)$ & $<0.001$ \\
\hline NAFLD fibrosis score & $-2.582(-3.366$ to -1.926$)$ & $-1.689(-2.770$ to -0.829$)$ & $<0.001$ \\
\hline Liver stiffness (kPa) & $5.2(4.2$ to 6.3$)$ & 7.6 (5.8 to 11.5$)$ & $<0.001$ \\
\hline
\end{tabular}

Values are presented as median (interquartile range).

NAFLD, nonalcoholic fatty liver disease; MAFLD, metabolic-associated fatty liver disease; MR, metabolic risk; APRI, aspartate aminotransferase to platelet ratio index; FIB-4, fibrosis-4.

in the fatty liver index, APRI, NAFLD fibrosis score, and liver stiffness, assessed using transient elastography, in MAFLD without NAFLD compared to non-MR NAFLD (Table 4), which might indicate that subjects with non-MR NAFLD might appear less severe than those with MAFLD, despite the comparable long-term implications of these diseases. These findings suggest that subjects with non-MR NAFLD might have no urgent diagnostic and therapeutic intervention needs, due to a potentially favorable disease course.

Although huge controversies still remain, the emphasis on metabolic dysfunction to define the subjects with MAFLD might underestimate the prognostic value of hepatic steatosis itself; therefore, it seems that subjects with non-MR NAFLD, a new disease category, who might have an intermediate risk of metabolic dysfunction when comparing subjects with neither NAFLD nor MAFLD and those with MAFLD, require clinical attention. In addition, varying metabolic phenotypes should be compared between subjects without NALFD or MALFD, those with non-MR NAFLD, and those with MAFLD. If feasible, histopathological interpretation or comparisons are warranted to elucidate the characteristics of non-MR NAFLD. Recently, a Taiwanese study using only histological information ${ }^{11}$ showed that $42.8 \%$ of the patients with MAFLD (67 of 157) presented with advanced liver fibrosis, while none of the nine subjects with non-MR NAFLD did.

\section{GENETICS}

The pathogenesis of NAFLD is complex and likely shaped by the dynamic interactions between genetic susceptibility and environmental factors. These factors interact with environmental, dietary, and metabolic risk factors, which contribute to the development of NAFLD and the risk of disease progression. Different genes encode proteins involved in the regulation of lipid metabolism in the liver. Over the past two decades, sequence variations in various genetic loci, including PNPLA3, TM6SF2, GCKR, MBOAT7, and HSD17B13, have been shown to increase NAFLD susceptibility. ${ }^{19}$ These genes can be used in clinical risk stratification and personalized therapy. However, the role of these genetic variations in patients with MAFLD, which is a newly proposed disease category, have not been fully investigated, and further evaluation is needed.

A recent study by Liu et al. ${ }^{20}$ reported the genotype data from 160,979 subjects in the UK Biobank, derived from a GWAS chip (Affymetrix UK BiLEVE and UK Biobank Axiom arrays). The impact of MAFLD, especially on hepatic events, was amplified by a high genetic risk score, of which genetic variations in PNPLA3, TM6SF2, and MBOAT7 play principal roles. However, the amplified effects of high genetic risk scores for each of these genes were not found in subjects with lean MAFLD, which was defined as MAFLD with a BMl $<25 \mathrm{~kg} / \mathrm{m}^{2}$.

A retrospective case-control study evaluated the association of single nucleotide polymorphisms with the risk of MAFLD in a Singaporean Chinese population and their interactions with environmental and medical risk factors. ${ }^{21}$ This study found that PNPLA3 GG was more likely to be associated with MAFLD (43.4\% CC vs. $69.7 \% \mathrm{GG}, P=0.017$ and $44.8 \%$ CG vs. $69.7 \% \mathrm{GG}, P=0.022$ ), while variations in LYPLAL1, GCKR, FDFT1, COL13A1, PZP, and TM6SF2 were not significantly associated with these patients.

The influence of genetic variation can be translated into more precise clinical management, which should be tailored to each individual patient with MAFLD.

\section{COMPARISON BETWEEN NAFLD AND MAFLD: CROSS-SECTIONAL CONSIDERATIONS}

Lin et al. ${ }^{22}$ recently reported real-world data comparing NAFLD and MAFLD (Table 5). They analyzed 13,083 subjects from the third NHANESs of the United States, and classified 4,087 (31.2\%) subjects with MAFLD and 4,347 (33.2\%) with NAFLD. When 
Table 5. Comparison of NAFLD and MAFLD criteria

\begin{tabular}{|c|c|c|c|c|c|c|}
\hline \multirow[b]{2}{*}{ Outcome } & \multirow{2}{*}{$\begin{array}{c}\text { NAFLD } \\
(n=4,347)\end{array}$} & \multirow{2}{*}{$\begin{array}{c}\text { MAFLD } \\
(n=4,087)\end{array}$} & \multirow{2}{*}{$\begin{array}{l}\text { Non-MR NAFLD } \\
\qquad(n=620)\end{array}$} & \multicolumn{3}{|c|}{$P$-value } \\
\hline & & & & $\begin{array}{l}\text { NAFLD vs. } \\
\text { MAFLD }\end{array}$ & $\begin{array}{c}\text { NAFLD vs. } \\
\text { non-MR NAFLD }\end{array}$ & $\begin{array}{c}\text { MAFLD vs. } \\
\text { non-MR NAFLD }\end{array}$ \\
\hline Age (years) & $46.81 \pm 15.77$ & $48.39 \pm 15.20$ & $35.13 \pm 13.44$ & $<0.001$ & $<0.001$ & $<0.001$ \\
\hline Male (\%) & 2,014 (46.33) & $2,036(49.82)$ & $249(40.16)$ & 0.001 & 0.004 & $<0.001$ \\
\hline BMI $\left(\mathrm{kg} / \mathrm{m}^{2}\right)$ & $29.49 \pm 6.69$ & $30.68 \pm 6.25$ & $21.67 \pm 2.08$ & $<0.001$ & $<0.001$ & $<0.001$ \\
\hline Diabetes (\%) & $1,092(25.12)$ & $1,171(28.65)$ & $0(0)$ & $<0.001$ & $<0.001$ & $<0.001$ \\
\hline Hypertension (\%) & 1,343 (30.89) & $1,463(35.80)$ & $26(4.19)$ & $<0.001$ & $<0.001$ & $<0.001$ \\
\hline $\mathrm{ALT}(\mathrm{IU} / \mathrm{L})$ & $22.31 \pm 21.34$ & $23.96 \pm 22.22$ & $16.81 \pm 17.84$ & $<0.001$ & $<0.001$ & $<0.001$ \\
\hline NFS score & $-2.18 \pm 1.52$ & $-2.05 \pm 1.51$ & $-3.00 \pm 1.32$ & $<0.001$ & $<0.001$ & $<0.001$ \\
\hline FIB-4 score & $1.01 \pm 0.84$ & $1.06 \pm 1.35$ & $0.87 \pm 1.05$ & 0.033 & 0.002 & $<0.001$ \\
\hline
\end{tabular}

Values are presented as mean \pm standard deviation or number $(\%)$.

NAFLD, nonalcoholic fatty liver disease; MAFLD, metabolic-associated fatty liver disease; MR, metabolic risk; BMI, body mass index; ALT, alanine aminotransferase; NFS, NAFLD fibrosis score; FIB-4, fibrosis-4.

these subjects were compared, they found that patients with MAFLD were significantly older and had higher BMI and higher proportions of metabolic comorbidities (T2DM, hypertension) compared to the subjects with NAFLD. Subjects with MAFLD and alcohol consumption were younger and had fewer metabolic disorders and a higher proportion of advanced fibrosis compared to those without. This data suggest that the MAFLD criteria are practical and provide physicians with the opportunity to treat patients holistically, rather than focusing on individual organ systems.

Yamamura et al. ${ }^{18}$ also reported that MAFLD was better at identifying subjects with significant liver fibrosis than NAFLD in their study of 765 Japanese subjects with FLD. Liver stiffness using shear wave elastography was significantly higher in MAFLD than in NAFLD (7.7 vs. $6.8 \mathrm{kPa}, P=0.001$ ). Using decision-tree analysis, MAFLD, without NAFLD and alcohol consumption, was the initial classifier for significant liver fibrosis.

In contrast, Wai-Sun Wong et al. ${ }^{23}$ reported the impact of MAFLD criteria on the epidemiology of FLD by analyzing 922 adults from the Hong Kong census database. The prevalence of NAFLD and MAFLD was $25.7 \%$ and $25.9 \%$, respectively, and the addition of the MAFLD criteria did not significantly change the prevalence of NAFLD. However, the incidence of MAFLD (2.8/100 person-years) was $25 \%$ lower than that of NAFLD (3.7/100 person-years). This difference was predominantly observed in subjects with a BMI of $<23 \mathrm{~kg} / \mathrm{m}^{2}$ (0.9 vs. 2.2/100 person-years). These data suggested that the new MAFLD criteria may exclude patients with a more benign clinical course.

\section{COMPARISON OF NAFLD AND MAFLD: LONGI- TUDINAL CONSIDERATIONS}

Although the prognostic significance of MAFLD has been investigated in various clinical settings, few studies have investigated the prognostic value of MAFLD in comparison with NAFLD. A recent community-based cohort study by Niriella et al. ${ }^{24}$ conducted in suburban Sri Lanka reported the findings for a study in which subjects were randomly selected in 2007 and then reassessed in 2014 to evaluate the new-onset metabolic traits and CVD events. In this study, among the 2,985 subjects recruited in 2007, 940 (31.5\%) had NAFLD, 990 (33.1\%) had MAFLD, and 362 (12.1\%) were controls. MAFLD captured an additional $2.9 \%$ and lost $1.3 \%$ of all study subjects when compared to NAFLD, and at baseline, the anthropometric and metabolic traits were similar between the two groups. At the 7-year follow-up, the risk of new-onset metabolic traits and fatal/non-fatal CVD events were similar in both groups, but significantly higher compared to the controls (Table 6). Those excluded by the NAFLD definition but captured by the MAFLD definition (MAFLD, but without NAFLD) showed higher baseline metabolic traits compared to those excluded by the MAFLD definition but captured by the NAFLD definition (non-MR NAFLD), and had a substantially higher risk of new-onset metabolic traits and CVD events compared to the controls. Therefore, these authors concluded that redefining NAFLD as MAFLD seemed to improve clinical utility.

Another nationwide cohort study from South Korea conducted by Lee et al. ${ }^{16}$ evaluated the prevalence of FLD, including MALFD 
Table 6. New-onset metabolic traits and CVD events

\begin{tabular}{|c|c|c|c|c|c|c|c|}
\hline \multirow[b]{2}{*}{ Outcome } & \multirow{2}{*}{$\begin{array}{l}\text { Control } \\
\left(n^{*}=255\right)\end{array}$} & \multirow{2}{*}{$\begin{array}{l}\text { NAFLD } \\
\left(n^{*}=708\right)\end{array}$} & \multicolumn{2}{|c|}{ NAFLD (vs. control) } & \multirow{2}{*}{$\begin{array}{l}\text { MAFLD } \\
\left(n^{*}=735\right)\end{array}$} & \multicolumn{2}{|c|}{ MAFLD (vs. control) } \\
\hline & & & $\begin{array}{l}\text { Adjusted RR } \\
(95 \% \mathrm{Cl})\end{array}$ & $P$-value & & $\begin{array}{l}\text { Adjusted RR } \\
(95 \% \mathrm{Cl})\end{array}$ & $P$-value \\
\hline Incident general obesity & $9 / 254(3.5 \%)$ & $51 / 215(24.6 \%)$ & $7.7(3.8-15.4)$ & $<0.001$ & $57 / 213(26.8 \%)$ & $8.3(4.1-16.6)$ & $<0.001$ \\
\hline Incident central obesity & 39/246 (15.9\%) & 37/109 (33.9\%) & $2.6(1.8-3.8)$ & $<0.001$ & 44/101 (43.6\%) & $3.3(2.3-4.7)$ & $<0.001$ \\
\hline Incident diabetes & $31 / 243(12.8 \%)$ & 203/503 (40.4\%) & $3.1(2.2-4.4)$ & $<0.001$ & 216/523 (41.3\%) & $3.2(2.3-4.5)$ & $<0.001$ \\
\hline Incident hypertension & $36 / 218(16.5 \%)$ & 109/232 (33.7\%) & $2.1(1.4-2.8)$ & $<0.001$ & $111 / 326(34.0 \%)$ & $2.1(1.5-2.9)$ & $<0.001$ \\
\hline $\begin{array}{l}\text { Incident } \\
\text { hypertriglyceridemia }\end{array}$ & 68/231 (29.4\%) & $145 / 361(40.2 \%)$ & $1.3(1.1-1.7)$ & 0.026 & 153/372 (41.1\%) & $1.4(1.1-1.7)$ & 0.010 \\
\hline $\begin{array}{l}\text { Incident hypo HDL } \\
\text { cholesterolemia }\end{array}$ & 68/208 (32.7\%) & $246 / 461(53.4 \%)$ & $1.5(1.2-1.7)$ & $<0.001$ & 250/487 (51.3\%) & $1.5(1.2-1.8)$ & $<0.001$ \\
\hline $\begin{array}{l}\text { CVD non-fatal and fatal } \\
\text { events }\end{array}$ & $4 / 253(1.6 \%)$ & $36 / 665$ (5.4\%) & $3.7(1.3-10.3)$ & 0.013 & 43/692 (6.2\%) & $4.2(1.5-11.5)$ & 0.006 \\
\hline
\end{tabular}

CVD, cardiovascular disease; NAFLD, nonalcoholic fatty liver disease; MAFLD, metabolic-associated fatty liver disease; RR, relative risk; $\mathrm{Cl}$, confidence interval; $\mathrm{HDL}$, high-density lipoprotein.

*The number in the original 2007 cohort irrespective of the presence or absence of baseline condition. Individuals with existing condition at baseline were excluded in the calculation of risk ratios.

Table 7. Cardiovascular disease risk according to the presence and combination of NAFLD and/or MAFLD

\begin{tabular}{lcccccc}
\hline \multirow{2}{*}{ Group } & Event & Person-years & Rate* & \multicolumn{2}{c}{ Hazard ratio (95\% confidence interval) } \\
\cline { 5 - 7 } & & & & Model $\mathbf{1}$ & Model 2 & Model 3 \\
\hline No NAFLD & 108,283 & $63,604,662$ & 170.2 & 1.00 (reference) & 1.00 (reference) & 1.00 (reference) \\
\hline NAFLD & 74,140 & $23,921,515$ & 309.9 & $1.82(1.80-1.84)$ & $1.40(1.38-1.41)$ & $1.41(1.40-1.43)$ \\
\hline No MAFLD & 81,235 & $55,715,210$ & 145.8 & 1.00 (reference) & 1.00 (reference) & 1.00 (reference) \\
\hline MAFLD & 101,188 & $31,810,967$ & 318.1 & $2.18(2.16-2.20)$ & $1.56(1.54-1.57)$ & $1.52(1.51-1.54)$ \\
\hline Neither FLD & 79,987 & $55,203,158$ & 144.9 & 1.00 (reference) & 1.00 (reference) & 1.00 (reference) \\
\hline Non-MR NAFLD & 1,248 & 512,052 & 243.7 & $1.68(1.59-1.78)$ & $1.20(1.13-1.27)$ & $1.09(1.03-1.15)$ \\
\hline MAFLD, but without NAFLD & 28,296 & $8,401,504$ & 336.8 & $2.33(2.30-2.36)$ & $1.55(1.52-1.57)$ & $1.43(1.41-1.45)$ \\
\hline Both FLD & 72,892 & $23,409,463$ & 311.4 & $2.15(2.13-2.17)$ & $1.57(1.55-1.58)$ & $1.56(1.54-1.58)$ \\
\hline
\end{tabular}

Model 1 was unadjusted; model 2 was adjusted for age and sex; and model 3 was further adjusted for household income quartile, residential area, Charlson comorbidity index, tobacco use, exercise frequency, and estimated glomerular filtration rate.

NAFLD, nonalcoholic fatty liver disease; MAFLD, metabolic-associated fatty liver disease; MR, metabolic risk; FLD, fatty liver disease.

*Per 100,000 person-years.

and NAFLD, and the associated CVD risk using each of these definitions. From a nationwide health screening database, a total of 9,584,399 subjects ( $48.5 \%$ male) aged $40-64$ years between 2009 and 2010 were included. These subjects were categorized by the presence of NAFLD and MAFLD, separately, and by the combination of the two definitions: neither-FLD, NAFLD-alone (non-MR NAFLD), MAFLD-alone (MAFLD, but without NALFD), or both-FLD. The prevalence of NAFLD and MAFLD were $28.0 \%$ and $37.3 \%$, respectively. The median follow-up period was 10.1 years. NAFLD and MAFLD were associated with a significantly higher risk for CVD events. When the neither-FLD group was the refer- ence, multivariable-adjusted HRs ( $95 \% \mathrm{Cl}$ ) for CVD events were 1.09 (1.03-1.15) in the NAFLD-alone group, $1.43(1.41-1.45)$ in the MAFLD-alone group, and 1.56 (1.54-1.58) in the both-FLD groups, indicating the highest risk of CVD in the MAFLD group (Table 7). Given this, the authors concluded that a considerable proportion of middle-aged Korean adults have MAFLD, without satisfying the former definition of NAFLD, and that the change from NAFLD to MAFLD criteria may identify a greater number of individuals with metabolically complicated fatty liver and increased risk for CVD. 


\section{CLINICAL IMPLICATIONS OF THE TERMINOL- OGY CHANGE}

Patients who are well-informed and understand their condition can actively collaborate with their doctor to improve self-care and their quality of life, especially those with chronic disease. ${ }^{25}$ There is a growing awareness of the weaknesses inherent in the term "non-alcoholic," which overemphasizes "alcohol." The term "nonalcoholic" leads to the question, "If my disease is not alcohol-related, then what is the real cause?" ${ }^{26}$ The traditional terminology of "NAFLD" not only confuses patients in terms of the real cause of their disease, but can also lead to adverse consequences in terms of doctor-patient communication.

Recently, the overestimation of the exclusion of alcohol has sparked some heated debate about the threshold for "significant" alcohol consumption for the diagnosis of NAFLD. Different definitions of "significant" alcohol consumption are also present in the universal guidelines. ${ }^{6,727}$ For example, the European Association for the Study of the Liver guideline suggests that the diagnosis of NAFLD requires the exclusion of daily alcohol consumption $\geq 30 \mathrm{~g}$ for men and $\geq 20 \mathrm{~g}$ for women. ${ }^{7}$ Both the American Association for the Study of Liver Diseases and the Asian Pacific Association for the Study of the Liver guidelines define heavy or at-risk drinking as more than 14 drinks per week for men or more than seven drinks per week for women. ${ }^{6,27}$ In addition, the potential (contradictory) role of alcohol consumption in these "non-alcohol" diseases has been repeatedly raised. A large study of 58,927 participants with NAFLD demonstrated that light or moderate alcohol consumption is associated with worsening of fibrosis when compared to no consumption, ${ }^{28}$ while another prospective cohort study of 9,559 participants indicated that modest alcohol consumption markedly, and perhaps synergistically, increases the risk of developing cirrhosis when combined with obesity. ${ }^{29}$ In addition, patients diagnosed with NAFLD may interpret the name "non-alcoholic" as a license to continue drinking. ${ }^{26}$

Trivialization is considered a form of stigma as a minimizing action in which the disease is perceived as easy to obtain or treat, and it might lead to adverse disease outcomes. ${ }^{30}$ This mainly arises when disease perceptions or diagnoses are confusing. In fact, subjects with NAFLD experience trivialization of the disease. Several studies have reported that more than $95 \%$ of subjects with suspected NAFLD are still unaware of having liver disease, and most subjects ( $>75 \%$ ) do not feel that they are at risk of developing NAFLD. ${ }^{31-33}$ A recent report of a semi-structured interview with 136 subjects with NAFLD showed that the subjects tended to trivialize their condition. ${ }^{34}$ Most subjects (>80\%) indicated that they were not concerned at all, while in comparison, $>90 \%$ of the subjects indicated that they would be very concerned if they were told they had either hepatitis C or diabetes. Notably, 92\% of the subjects expressed major dissatisfaction with the term "NAFLD," mainly as it included the term "alcohol" in its name. While $72.8 \%$ of the subjects said they preferred the term "MAFLD," $5.8 \%$ indicated that they did not prefer this change. Moreover, a recent survey with 191 physicians presented that $96 \%$ of the subjects had a substantial lack of knowledge regarding the differences between NAFLD and NASH, even though they were aware of both. ${ }^{35}$ Therefore, changing the name is expected to decrease trivialization and increase awareness of the disease, leading patients to recognize the importance and effectiveness of critical lifestyle changes necessary to mitigate disease progression.

The term "non-alcoholic" underemphasizes the importance of metabolic dysfunction. A reorientation of the disease name to MAFLD, which includes the term "metabolic," may lead to better patient-to-physician communication and improve decision sharing by reducing the patients' confusion about the real cause of their disease. In addition, it is expected to help engage more healthcare systems and establish collaboration with other populations with increased metabolic risks (e.g., diabetes, renal disease, and CVD). ${ }^{36}$ Also, using the word "metabolic" in the context of the acronym hinges on the fact that MAFLD is potentially preventable and emphasizes the need for appropriate resource allocation and effective public health policy decisions. Changing the terminology has significant implications for the pharmaceutical industry, such as stimulating shared funding with other metabolic diseases and implementing effective system-wide interventions.

\section{FUTURE DIRECTIONS}

Part of the terminology change for MAFLD involves removing the term "non-alcoholic steatohepatitis" as a distinct subtype, and instead adopting a more fluid description based on grade and stage. MAFLD is viewed as a disease process in which simple steatosis (MAFLD with no inflammation and fibrosis) merges into MAFLD with; for example, grade 1 inflammation and stage 2 fibrosis to MAFLD with grade 0 inflammation and stage 4 fibrosis. ${ }^{37}$ Notably, previous studies of biopsy-proven NAFLD showed that the presence of NASH did not increase the risk of liver-specific morbidity or overall mortality. However, abandoning the term "steatohepatitis" could derail the ongoing clinical trials that were 
designed in concert with the guidance around NASH drug development. ${ }^{38}$ In this same context, some doubts have been raised as to whether now is the right time to change the name. It cannot be concluded that the terminology change will result in a new understanding of the disease. Therefore, some experts say it is not yet the time to impose another inappropriate term that needs to be corrected, as the understanding of the natural history, etiology, and management of NAFLD continues to be improved. ${ }^{38,39}$

NAFLD still contains a wide variety of disorders, and metabolic syndromes do form part of its spectrum (probably a major part) as a relatively sterile therapeutic armamentarium. Moreover, there are concerns that this may paradoxically result in misleading treatments for the concomitant metabolic disorders. However, NAFLD is a dynamic disease characterized by several factors that can change over time. Therefore, multiple phenotypic measurements still need to be further analyzed as separate components, reflecting the complex spectrum of this disease. Also, further clarification and stratification of MAFLD are needed to guide decisionmaking and prognostication from a clinical perspective.

\section{SUMMARY AND CONCLUSIONS}

Two recent studies have suggested that the MAFLD criteria can identify patients with a higher degree of disease severity. ${ }^{11,16}$ In contrast, another study showed that the MAFLD criteria might overlook the clinical and prognostic significance of non-MR NAFLD. ${ }^{16}$ Notably, several recent studies have shown that the clinical characteristics, including renal function and fibrosis markers, of subjects with non-MR NAFLD might be better than those of subjects with MAFLD. ${ }^{17}$ However, another study showed that subjects with non-MR NAFLD had a higher risk of CVD compared to those with neither NAFLD nor MAFLD, indicating the need for careful assessment and monitoring of this population. ${ }^{16}$ In addition, more studies are required to better elucidate the potential risk that can be the cause of future disease in the "lean" NAFLD subjects without any metabolic dysregulation who are excluded from the MAFLD criteria.

The role of genetic variation among subjects with MAFLD has not been fully investigated. Although one study indicated that PNPLA3 GG was more likely to be associated with MAFLD, ${ }^{40}$ the influence of genetic variation should be investigated and tailored to each subject. The MAFLD criteria seem to better identify subjects at higher risk of liver-related complications, as documented in a recent cross-sectional study. In addition, two large-sized co- hort studies with a median follow-up period of up to 10 years showed that the change from NAFLD to MAFLD criteria might identify a greater number of subjects with metabolically complicated FLD and increased risk for CVD. ${ }^{16,23}$ However, large followup studies are needed to compare the risk of developing important extra-hepatic outcomes (e.g., hepatocellular carcinoma or other malignancy) of patients with either MAFLD or NAFLD.

Due to the scarcity and controversy of the existing data, it still remains unclear whether NAFLD or MAFLD is more feasible in clinical practice. More studies comparing the NAFLD and MAFLD criteria and stratifying subjects with MAFLD into different risk groups are needed to draw firm conclusions.

\section{Authors' contributions}

Seong Hee Kang, Yuri Cho, Soung Won Jeong, Seung Up Kim and Jin-Woo Lee contributed to the literature review and manuscript preparation.

\section{Acknowledgements}

This study was supported by Basic Science Research Program through the National Research Foundation of Korea (NRF) funded by the Ministry of Science, ICT \& Future Planning (2018R1C1B5044890, 2019R1A2C4070136, 2020R1F1A1076282 and 2021R1A2C4001401) and the Soonchunhyang University Research Fund. The funders had no role in study design, data collection and analysis, decision to publish, or preparation of the manuscript.

This manuscript was reviewed by native speakers for English proof readings (Editage; certificate, YUCMR_5139).

\section{Conflicts of Interest}

Seung Up Kim has served as an advisory committee member of Gilead Sciences, Bayer, Eisai, and Novo Nordisk. He is a speaker for Gilead Sciences, GSK, Bayer, Eisai, Abbvie, EchoSens, MSD, Eisai, Otsuka, and Bristol-Myers Squibb. He has also received a research grant from Abbvie and Bristol-Myers Squibb. Jin-Woo Lee has served as an advisory committee member of Gilead Sciences, Abbvie and Novo Nordisk. The other authors declare that they have no competing interests.

\section{REFERENCES}

1. Schaffner $F$, Thaler $H$. Nonalcoholic fatty liver disease. Prog Liver Dis 1986;8:283-298. 
2. Eslam M, Sanyal AJ, George J; International Consensus Panel. MAFLD: a consensus-driven proposed nomenclature for metabolic associated fatty liver disease. Gastroenterology 2020;158:19992014.e1.

3. Cotter TG, Rinella M. Nonalcoholic fatty liver disease 2020: the state of the disease. Gastroenterology 2020;158:1851-1864.

4. Sookoian S, Pirola CJ. Precision medicine in nonalcoholic fatty liver disease: new therapeutic insights from genetics and systems biology. Clin Mol Hepatol 2020;26:461-475.

5. Yoo JJ, Kim W, Kim MY, Jun DW, Kim SG, Yeon JE, et al. Recent research trends and updates on nonalcoholic fatty liver disease. Clin Mol Hepatol 2019;25:1-11.

6. Chalasani N, Younossi Z, Lavine JE, Charlton M, Cusi K, Rinella M, et al. The diagnosis and management of nonalcoholic fatty liver disease: practice guidance from the American Association for the Study of Liver Diseases. Hepatology 2018;67:328-357.

7. European Association for the Study of the Liver (EASL); European Association for the Study of Diabetes (EASD); European Association for the Study of Obesity (EASO). EASL-EASD-EASO clinical practice guidelines for the management of non-alcoholic fatty liver disease. J Hepatol 2016;64:1388-1402.

8. Eslam M, Newsome PN, Sarin SK, Anstee QM, Targher G, RomeroGomez $M$, et al. A new definition for metabolic dysfunctionassociated fatty liver disease: an international expert consensus statement. J Hepatol 2020;73:202-209.

9. Sun DQ, Zheng KI, Xu G, Ma HL, Zhang HY, Pan XY, et al. PNPLA3 rs738409 is associated with renal glomerular and tubular injury in NAFLD patients with persistently normal ALT levels. Liver Int 2020; 40:107-119.

10. Zheng KI, Sun DQ, Jin Y, Zhu PW, Zheng MH. Clinical utility of the MAFLD definition. J Hepatol 2021;74:989-991.

11. Huang SC, Su HJ, Kao JH, Tseng TC, Yang HC, Su TH, et al. Clinical and histologic features of patients with biopsy-proven metabolic dysfunction-associated fatty liver disease. Gut Liver. 2021 Jan 12. doi: 10.5009/gnl20218.

12. Ciardullo S, Perseghin G. Prevalence of NAFLD, MAFLD and associated advanced fibrosis in the contemporary United States population. Liver Int. 2021 Feb 16. doi: 10.1111/liv.14828.

13. Huang J, Kumar R, Wang M, Zhu Y, Lin S. MAFLD criteria overlooks a number of patients with severe steatosis: is it clinically relevant? J Hepatol 2020;73:1265-1267.

14. Brunner KT, Pedley A, Massaro JM, Hoffmann U, Benjamin EJ, Long MT. Increasing liver fat is associated with progression of cardiovascular risk factors. Liver Int 2020;40:1339-1343.

15. Mantovani A, Petracca G, Beatrice G, Tilg H, Byrne CD, Targher G. Non-alcoholic fatty liver disease and risk of incident diabetes mellitus: an updated meta-analysis of 501022 adult individuals. Gut. 2020 Sep 16. doi: 10.1136/gutjnl-2020-322572.
16. Lee H, Lee YH, Kim SU, Kim HC. Metabolic dysfunction-associated fatty liver disease and incident cardiovascular disease risk: a nationwide cohort study. Clin Gastroenterol Hepatol. 2020 Dec 22. doi: 10.1016/j.cgh.2020.12.022.

17. Sun DQ, Jin Y, Wang TY, Zheng KI, Rios RS, Zhang HY, et al. MAFLD and risk of CKD. Metabolism 2021;115:154433.

18. Yamamura S, Eslam M, Kawaguchi T, Tsutsumi T, Nakano D, Yoshinaga $S$, et al. MAFLD identifies patients with significant hepatic fibrosis better than NAFLD. Liver Int 2020;40:3018-3030.

19. Lin YC, Wu CC, Ni YH. New perspectives on genetic prediction for pediatric metabolic associated fatty liver disease. Front Pediatr 2020;8:603654.

20. Liu Z, Suo C, Shi O, Lin C, Zhao R, Yuan H, et al. The health impact of MAFLD, a novel disease cluster of NAFLD, is amplified by the integrated effect of fatty liver disease-related genetic variants. Clin Gastroenterol Hepatol. 2020 Dec 30. doi: 10.1016/j.cgh.2020.12.033.

21. Kim KS, Lee BW. Beneficial effect of anti-diabetic drugs for nonalcoholic fatty liver disease. Clin Mol Hepatol 2020;26:430-443.

22. Lin S, Huang J, Wang M, Kumar R, Liu Y, Liu S, et al. Comparison of MAFLD and NAFLD diagnostic criteria in real world. Liver Int 2020;40:2082-2089.

23. Wai-Sun Wong V, Lai-Hung Wong G, Woo J, Abrigo JM, Ka-Man Chan C, She-Ting Shu S, et al. Impact of the new definition of metabolic associated fatty liver disease on the epidemiology of the disease. Clin Gastroenterol Hepatol. 2020 Oct 31. doi: 10.1016/ j.cgh.2020.10.046.

24. Niriella MA, Ediriweera DS, Kasturiratne A, De Silva ST, Dassanayaka AS, De Silva AP, et al. Outcomes of NAFLD and MAFLD: results from a community-based, prospective cohort study. PLoS One 2021;16:e0245762.

25. Vahdat S, Hamzehgardeshi L, Hessam S, Hamzehgardeshi Z. Patient involvement in health care decision making: a review. Iran Red Crescent Med J 2014;16:e12454.

26. Yilmaz Y, Byrne CD, Musso G. A single-letter change in an acronym: signals, reasons, promises, challenges, and steps ahead for moving from NAFLD to MAFLD. Expert Rev Gastroenterol Hepatol. 2020 Dec 9. doi: 10.1080/17474124.2021.1860019.

27. Wong VW, Chan WK, Chitturi S, Chawla Y, Dan YY, Duseja A, et al. Asia-Pacific working party on non-alcoholic fatty liver disease guidelines 2017-part 1: definition, risk factors and assessment. J Gastroenterol Hepatol 2018;33:70-85.

28. Chang Y, Cho YK, Kim Y, Sung E, Ahn J, Jung HS, et al. Nonheavy drinking and worsening of noninvasive fibrosis markers in nonalcoholic fatty liver disease: a cohort study. Hepatology 2019;69:64-75.

29. Liu B, Balkwill A, Reeves G, Beral V; Million Women Study Collaborators. Body mass index and risk of liver cirrhosis in middle aged UK women: prospective study. BMJ 2010;340:c912.

30. Robinson P, Turk D, Jilka S, Cella M. Measuring attitudes towards 
mental health using social media: investigating stigma and trivialisation. Soc Psychiatry Psychiatr Epidemiol 2019;54:51-58.

31. Cleveland ER, Ning H, Vos MB, Lewis CE, Rinella ME, Carr JJ, et al. Low awareness of nonalcoholic fatty liver disease in a populationbased cohort sample: the CARDIA study. J Gen Intern Med 2019;34: 2772-2778.

32. Singh A, Dhaliwal AS, Singh S, Kumar A, Lopez R, Gupta M, et al. Awareness of nonalcoholic fatty liver disease is increasing but remains very low in a representative US cohort. Dig Dis Sci 2020;65:978-986.

33. Wieland AC, Mettler P, MCDermott MT, Crane LA, Cicutto LC, Bambha KM. Low awareness of nonalcoholic fatty liver disease among patients at high metabolic risk. J Clin Gastroenterol 2015;49:e6-e10.

34. Alem SA, Gaber Y, AbdAlla M, Said E, Fouad Y. Capturing patient experience: a qualitative study of change from NAFLD to MAFLD real-time feedback. J Hepatol. 2021 Jan 23. doi: 10.1016/ j.jhep.2021.01.022.

35. Fouad Y, Gomaa A, Semida N, Ghany WA, Attia D. Change from NAFLD to MAFLD increases the awareness of fatty liver disease in primary care physicians and specialists. J Hepatol. 2021 Feb 11. doi: 10.1016/j.jhep.2020.12.035.

36. Shiha G, Korenjak M, Eskridge W, Casanovas T, Velez-Moller P, Högström $S$, et al. Redefining fatty liver disease: an international patient perspective. Lancet Gastroenterol Hepatol 2021;6:73-79.

37. Zheng KI, Fan JG, Shi JP, Wong VW, Eslam M, George J, et al. From NAFLD to MAFLD: a "redefining" moment for fatty liver disease. Chin Med J (Engl) 2020;133:2271-2273.

38. Younossi ZM, Rinella ME, Sanyal AJ, Harrison SA, Brunt EM, Goodman Z, et al. From NAFLD to MAFLD: implications of a premature change in terminology. Hepatology 2021;73:1194-1198.

39. Singh SP, Anirvan P, Reddy KR, Conjeevaram HS, Marchesini G, Rinella ME, et al. Non-alcoholic fatty liver disease: not time for an obituary just yet! J Hepatol 2021;74:972-974.

40. Lee GH, Phyo WW, Loo WM, Kwok R, Ahmed T, Shabbir A, et al. Validation of genetic variants associated with metabolic dysfunction-associated fatty liver disease in an ethnic Chinese population. World J Hepatol 2020;12:1228-1238. 